\title{
Morphological and electrical characterization of hybrid thin-film composed of titania nanocrystals, Poly (3-hexylthiophene) and Piper Betle Linn
}

\begin{abstract}
In this research, the effect of scan numbers of titania nanocrystals ( $\mathrm{TiO} 2 \mathrm{NCs}$ ) on the morphological and electrical characteristics of hybrid thin-films is investigated. These hybrid thin-films consist of a combination of organic (Piper Betle Linn extraction and Poly (3hexytlthiophene) (P3HT)) and inorganic $\mathrm{TiO} 2 \mathrm{NCs}$ (anatase structure) materials. These hybrid thin-films are fabricated in bilayer heterojunction of ITO/TiO2 NCs/P3HT/Piper Betle Linn via electrochemistry method using Electrochemical Impedance Spectroscopy (EIS). The scan numbers of TiO2 NCs are varied by 1, 3 and 5 number of scans. The morphological characterization is carried out via Field Emission Scanning Electron Microscopy (FESEM) meanwhile the electrical characteristic of the hybrid thin-film is measured by using four point probes. FESEM image indicates the particle size was found to be around 17-34 $\mathrm{nm}$. The increment of scan number of $\mathrm{TiO} 2 \mathrm{NCs}$ from one to five scan numbers of $\mathrm{TiO} 2 \mathrm{NCs}$ in bilayers thin films showed that the atomic percentage of titanium decrease from $5.23 \%$ to $2.20 \%$. This result indicates that as the thickness of thin films increases, the electrons required more energy to excite into conduction band of TiO2. Meanwhile, the electrical conductivities of hybrid solar cell increase from $0.385 \mathrm{Scm}-1$ to $0.389 \mathrm{Scm}-1$ as the scan numbers of $\mathrm{TiO} 2$ increase from one to three, however the electrical conductivity decrease to $0.346 \mathrm{Scm}-1$ at five scan numbers. As a conclusion, this study shows that the morphological and electrical properties of hybrid thin-films can be significantly affected by the scan number of $\mathrm{TiO} 2 \mathrm{NCs}$.
\end{abstract}

Keyword: Electrical conductivity; Field Emission Scanning Electron Microscopy; Piper Betle Linn; Poly (3-hexylthiophene); Titania nanocrystals 